\title{
What potential does plasmonics-amplified synergistic immuno photothermal nanotherapy have for treatment of cancer?
}

\author{
Tuan Vo-Dinh ${ }^{*}, 1,2,3$ \& Brant A Inman $3,4,5$ \\ ${ }^{1}$ Department of Biomedical Engineering, Duke University, Durham, NC 27710, USA \\ ${ }^{2}$ Department of Chemistry, Duke University, Durham, NC 27710, USA \\ ${ }^{3}$ Fitzpatrick Institute of Photonics, Duke University, Durham, NC 27710, USA \\ ${ }^{4}$ Division of Urology, Duke University, Durham, NC 27710, USA \\ ${ }^{5}$ Duke Cancer Institute, Duke University, Durham, NC 27710, USA \\ * Author for correspondence: tuan.vodinh@duke.edu
}

"Our results have indicated that by using a combination of immune-checkpoint inhibition and gold nanostar (GNS)-mediated photothermal therapy, it is possible to achieve complete eradication of primary treated tumors as well as distant untreated tumors tumors and induce long-term anti-cancer immunity in mice implanted with the MB49 bladder cancer cell line."

First draft submitted: 8 November 2017; Accepted for publication: 24 November 2017; Published online: 11 December 2017

Keywords: cancer therapy $\bullet$ hyperthermia $\bullet$ immune checkpoint receptor $\bullet$ immunotherapy $\bullet$ nanomedicine $\bullet$ nanotechnology • nanotherapy • photothermal treatment • plasmonic • surface-enhanced Raman scattering (SERS)

The war on cancer was declared almost half a century ago and yet the incidence of cancer is decreasing for only a handful of cancer types and most cancers that have spread beyond their organ of origin remain incurable. In fact, cancer is the most common cause of death in people aged less than 85 years and it is estimated that in 2012 there were more than 14.1 million new cancer cases worldwide and 8.2 million deaths that resulted [1].

Nanomedicine has contributed to important advances in healthcare over the past few decades. In particular, the use of nanoparticles in medicine has attracted increased attention for their unique efficacy and specificity in therapy [2]. One exciting new development is nanoparticle-mediated thermal therapy which has demonstrated the potential for precise tumor cell ablation [3], radio-sensitization of hypoxic regions [4], enhancement of drug delivery [5], activation of thermosensitive agents [6] and enhancement of the immune system [7]. A special type of metallic nanoparticles, called 'plasmonic' nanoparticles, has received great interest because they exhibit enhanced optical and electromagnetic properties. Our fundamental studies of various plasmonic structures [8-10] and applied development of plasmonic nanoplatforms have led to a wide variety of applications ranging from chemical sensing [10-12] to cancer diagnostics [13-17] and therapy using nanoparticle-mediated photothermal treatment [15,18-20]. Recently, we demonstrated that the use of plasmonic gold nanostars (GNS) in combination with immunotherapy - a treatment we referred to as Synergistic Immuno Photothermal Nanotherapy (SYMPHONY) - can dramatically enhance the efficacy of immunotherapy. Remarkably, we have found that SYMPHONY not only eradicates primary 'treated' tumors but also has resulted in the immune-mediated destruction of distant 'untreated' metastatic tumors [21]. This abscopal effect occurred uniquely when nanoparticle-mediated photothermal heating of tumors was used in combination with immunotherapy.

Plasmonic nanoparticles have unique properties that allow them to amplify the optical properties of the excitation light and thus increase the effectiveness of light-based photothermal tumor ablation. The unique properties which contribute to plasmonics-amplified immune nanotherapy for cancer, include: plasmonic nano-enhancers of light, nano-targeting of tumor cells, nanosources for heating tumor cells from the inside, nano-activators of the immune system and synergistic amplification of immunomodulation. These elements are discussed in the following sections.

Future Medicine 


\section{Plasmonic nano-enhancers of light}

Under incident electromagnetic field irradiation (e.g., a laser beam), conduction electrons in metallic nanoparticles are displaced into frequency oscillation equal to the incident light. These oscillating electrons are called surface plasmons - hence the term plasmonic nanoparticles - and produce a secondary electric field which adds to the incident light field. This condition yields intense localized energy fields that are concentrated at high curvature points on nanoparticles, a perfect physical property to exploit for photothermal tissue heating. Among nanoparticles used for light-induced photothermal therapy, GNS are of particular interest because their structure has multiple sharp branches that produce the numerous curvatures responsible for the 'lightning rod' effect that strongly enhances the local electromagnetic field when subject to light stimulation [22,23]. Theoretical calculations of the magnitude of the electric field indicate that the largest e-field enhancement occurs at the tips of the branches of the star [24]. Furthermore, GNS offer optical tunability that can be engineered by making subtle changes in their synthesis chemistry that results in geometry changes that tune their peak absorption band into the near infrared $(700-1200 \mathrm{~nm})$ wavelength. Exciting GNS with near infrared light is ideal for biological applications because the wavelengths are within the optical window where tissue absorption is minimized $[20,23]$ and thus photons can travel further through the tissues to be captured and converted into heat by GNS accumulating within a tumor [18].

With the goal of developing biocompatible nanoparticles for in vivo applications, our group first introduced a surfactant-free synthesis chemistry that does not require toxic cetyltrimethylammonium bromide for producing GNS [23]. GNS are exceptional light 'nano-enhancers' that have led to a wide range of chemical, biological and medical (both diagnostic and therapeutic) applications. For example, GNS exhibit intense surface-enhanced Raman scattering and two-photon luminescence and therefore provide an exceptional platform for nucleic acid biomarker detection [13,17], stem cell tracking [25,26] and tumor bioimaging [18,19].

\section{Nano-targeting tumor cells}

As with other nanoparticles, GNS sizes can be controlled so that they passively accumulate in tumors due to the enhanced permeability and retention (EPR) effect of tumor vasculature. The EPR effect is a result of the inherent leakiness of the tumor vasculature which is underdeveloped and allows nanoparticles to escape the circulation and accumulate passively in tumors. In addition, retention of nanoparticles in the tumor is enhanced by the lack of an efficient lymphatic system which would normally carry extravasated fluid back to the circulation. To take advantage of the EPR effect, nanoparticles must be designed to have a narrow size range between approximately 10 and $100 \mathrm{~nm}$. Nanoparticles with sizes smaller than $10 \mathrm{~nm}$ are rapidly cleared by the kidneys [27] and those with sizes larger than $100 \mathrm{~nm}$ are cleared by the reticuloendothelial system [28]. GNS take advantage of the EPR effect because they can be synthesized to have hydrodynamic sizes that fit well in the 10-100 nm size range. GNS can even be used to target sites that are traditionally very difficult to access with drugs like the brain. Indeed, we have demonstrated in mouse models of brain cancer that we were able to achieve selective accumulation of GNS within target brain tumors but not in adjacent normal brain tissue, demonstrating an unprecedented spatial specificity for focal disruption of brain tumor vasculature and blood-brain barrier [29].

\section{Nanosources for heating tumor cells 'from the inside"}

Therapeutic hyperthermia has been used to treat cancer for many years and consists of the application of heat to a tumor or organ [30]. By elevating the temperature to more than $42^{\circ} \mathrm{C}$, malignant cells are killed through apoptosis or necrosis. Traditional therapeutic hyperthermia modalities, such as microwave or radiofrequency, and focused ultrasound have been used to control macroscopic heating around the tumor region, but cannot target or ablate cancer cells at the microprecision scale. Nanoparticle-mediated thermal therapy has recently received increasing interest [3,18-20,31-35]. GNS, which accumulate in and around cancer cells, can be triggered with light to rapidly achieve high ablative intratumoral temperatures $\left(>55^{\circ} \mathrm{C}\right)$ and can also induce milder fever-range (41$43^{\circ} \mathrm{C}$ ) hyperthermia in the tumor microenvironment. The combination of the EPR effect and the capacity for efficient photon to heat conversion, make GNS an ideal photothermal transducer for selective cancer therapy at the nanoscale level, as we have demonstrated both in vitro and in vivo [18-20]. Theoretical calculations have shown that GNS have the highest absorption-to-scattering ratio of the commonly used plasmonic gold nanoparticles, which is consistent with the very high conversion efficiency we have observed [24]. GNS therefore can act as nanosources of heat that can ablate tumor cells in their microenvironment (i.e., from the inside) with high spatial accuracy. This feature improves tumor-targeting precision and permits the use of reduced laser energy required to destroy the 
targeted cancer cells. The ability to selectively heat tumor areas where GNS are located while keeping surrounding healthy tissues at significantly lower temperatures offers significant advantages over other thermal therapies.

\section{Nano-activators of the immune system}

Ablative tumor treatments, such as hyperthermia, photodynamic therapy and cryoablation, can induce strong tumor-specific immune responses [36,37]. Similarly, plasmonic nanoparticles such as GNS operate as nano-activators of the immune system in two ways [21]. First, following ablation, dying tumor cells release tumor-specific antigens, damage-associated molecular pattern molecules (DAMPs), and heat shock proteins. In live cells, DAMPs are intracellular molecules that are normally hidden. When cells are damaged or dying, DAMPS that are released demonstrate immunostimulatory properties. For example, DAMPS have been shown to exert various effects on antigen-presenting cells, such as promoting maturation, activation and antigen processing/presentation [38]. Antigen-presenting cells, which are present in the tissue or in local draining lymph nodes, process the tumor antigens and present tumor-derived peptides to $\mathrm{T}$ cells, triggering the immune response. Second, photothermal treatment can also create fever-like temperatures, which have been shown to extend and enhance the effects of various immunotherapies by activating and stimulating innate and adaptive immune responses and improving tumor blood flow [39]. The immune system activation is triggered and heightened by several temperature-induced mechanisms, improved leukocyte trafficking to the tumor, better antigen presentation and better leukocyte activation. All of these provide a staging ground for immune defense against cancer [36,40].

\section{Synergistic nano-amplifiers of immunomodulation}

A variety of strategies have been used to treat cancer (e.g., chemotherapy, radiotherapy), but currently immunotherapy is experiencing a resurgence. In particular, drugs targeting immune checkpoints have recently shown outstanding efficacy in the treatment of cancer [40]. The immune checkpoints are normally safeguards used by the body to prevent inappropriate or overactivation of the immune response. However, many tumors have acquired the ability to manipulate these checkpoints to switch the immune system into a state of unresponsiveness. Because immune checkpoints consist of ligand-receptor interactions, they can be readily blocked by antibodies or modulated by ligands or receptors [41]. The CTLA-4, the first immune checkpoint receptor to be clinically targeted, is expressed exclusively on $\mathrm{T}$ cells where it primarily regulates the amplitude of the early stages of T-cell activation. Another immune-checkpoint receptor, PD-1, is emerging as a promising target. The two ligands for PD-1 are PD-L1 (also known as B7-H1 and CD274) and PD-L2 (also known as B7-DC and CD273). PD-L1 and PD-L2 also contribute to the suppression of the immune system [42-45]. PD-L1 binds to the PD-1 receptor found on activated T cells and inhibits cytotoxic T-cell function, thus hindering the immune response. Drugs that block the interaction between PD-1 and PD-L1 can result in enhancement of T-cell activation and effector functions resulting in a swift tissue inflammatory response. However, checkpoint-based drugs work only for a limited number of patients and can become ineffective with time. Broadening and stabilizing the effect of PD-L1/PD1 inhibitors can be achieved with nanoparticle-mediated synergistic thermal therapies [21]. Our results have indicated that by using a combination of immune-checkpoint inhibition and gold nanostar (GNS)-mediated photothermal therapy, it is possible to achieve complete eradication of primary treated tumors as well as distant untreated tumors tumors and induce long-term anti-cancer immunity in mice implanted with the MB49 bladder cancer cell line. Immunotherapies could thus synergistically benefit from targeted thermal nanotherapies, especially when hyperthermia around immune-checkpoint inhibitors in the tumor bed is combined with precise thermal ablation of cancer cells [46]. The effectiveness of the combination of plasmonic GNS-enabled photothermal ablation and PD-L1 immunomodulation was demonstrated to be synergistic (not just additive) and delayed rechallenge with repeated MB49 tumor injections did not lead to new tumor formation, indicating that the combined treatment induced effective long-lasting immunity.

\section{Conclusion}

Our studies of plasmonic nanoparticles, such as GNS, have demonstrated their effectiveness in laser light enhancing, in selective targeting of tumor cells, in photothermal treatment and in activating the immune response for cancer therapy. Synergistic combinatorial approaches such as SYMPHONY have great potential to reverse tumor-mediated immunosuppression in order improve cancer immunotherapy outcomes. This effective integration of the latest advances in nanotechnology and immunotherapy shows great promise to treat not only unresectable primary tumors, but also distant cancer metastasis by enhancing the systemic activity of specific and adaptive immune responses. Of great importance is the possibility that such an approach can induce long-term immunological 
memory that can provide protection against tumor recurrence long after treatment of the initial tumors. In other words, SYMPHONY works like both a primary ablative treatment and a vaccine simultaneously. Further fundamental studies will achieve better understanding and optimal exploitation of the mechanisms underlying these novel synergistic treatment modalities in order to enhance and broaden the effect of immune-checkpoint inhibitors for successful eradication of metastatic cancer. Identifying and understanding the specific immune cells and molecular processes involved in this synergistic interaction will pave the way for successful treatment of locally advanced and metastatic cancer. The proposed synergistic immuno-nanotherapy approach could provide an effective treatment when aggressive tumors cannot be surgically removed and eventually spread throughout the body. The US FDA approval for use in humans of the CTLA-4 and PD-L1-based immunotherapies will make the drugs readily available for combination clinical trials. Similarly, as gold nanoparticles are considered biologically inert (nontoxic) and FDA-approved lasers are currently available for clinical use, the path to prove the safety of synergistic therapies for use in humans could be conceivable in a reasonable future. This strategy could lead to an entirely new treatment paradigm that challenges traditional surgical resection approaches for many cancers and metastases.

Financial \& competing interests disclosure

This work was supported by the Department of Defense (W81XWH-17-1-0567). The authors have no other relevant affiliations or financial involvement with any organization or entity with a financial interest in or financial conflict with the subject matter or materials discussed in the manuscript apart from those disclosed.

No writing assistance was utilized in the production of this manuscript.

\section{References}

1. World Health Organization. Latest world cancer statistics: global cancer burden rises to 14.1 million new cases in 2012: marked increase in breast cancers must be addressed. Press Release No. 223 (2013). www.iarc.fr/en/media-centre/pr/2013/pdfs/pr223_E.pdf

2. He Q, Guo S, Qian S, Chen X. Development of individualized anti-metastasis strategies by engineering nanomedicines. Chem. Soc. Rev. $44,6258-6286$ (2015).

3. Loo C, Lin A, Hirsch L et al. Nanoshell-enabled photonics-based imaging and therapy of cancer. Technology Cancer Res. Treat. 3(1), 33-40 (2004).

4. Pandita TK, Pandita S, Bhaumik SR. Molecular parameters of hyperthermia for radiosensitization. Crit. Rev. Eukaryot. Gene Exp. 19(3), 235-251 (2009).

5. Takada T, Yamashita T, Sato M et al. Growth inhibition of re-challenge B16 melanoma transplant by conjugates of melanogenesis substrate and magnetite nanoparticles as the basis for developing melanoma-targeted chemo-thermo-immunotherapy. J. Biomed. Biotechnol. 2009, 457936 (2009).

6. Koning GA, Eggermont AMM, Lindner LH, ten Hagen TLM. Hyperthermia and thermosensitive liposomes for improved delivery of chemotherapeutic drugs to solid tumors. Pharm. Res. 27(8), 1750-1754 (2010).

7. Wang C, Xu L, Liang C, Xiang J, Peng R, Liu Z. Immunological responses triggered by photothermal therapy with carbon nanotubes in combination with anti-CTLA-4 therapy to inhibit cancer metastasis. Adv. Mater. 26(48), 8154-8162 (2014).

8. Dhawan A, Norton SJ, Gerhold MD, Vo-Dinh T. Comparison of FDTD numerical computations and analytical multipole expansion method for plasmonics-active nanosphere dimers. Opt. Express 17, 9688-9703 (2009).

9. Khoury CG, Norton SJ, Vo-Dinh T. Plasmonics of 3-D nanoshell dimers using multipole expansion and finite element method. ACS Nano 3, 2776-2788 (2009).

10. Vo-Dinh T, Dhawan A, Norton SJ et al. Plasmonic nanoparticles and nanowires: design, fabrication and application in sensing. J. Phys. Chem. C Nanomater. Interfaces 114, 7480-7488 (2010).

11. Yuan H, Fales AM, Khoury CG, Liu Y, Vo-Dinh T. Spectral characterization and intracellular detection of surface-enhanced Raman scattering (SERS)-encoded plasmonic gold nanostars. J. Raman Spectrosc. 44, 234-239 (2013).

12. Bello JM, Narayanan VA, Stokes DL, Vo-Dinh T. Fiber-optic remote sensor for in situ surface-enhanced Raman scattering analysis. Anal. Chem. 62, 2437-2441 (1990).

13. Wang HN, Vo-Dinh T. Multiplex detection of breast cancer biomarkers using plasmonic molecular sentinel nanoprobes. Nanotechnology 20, 065101 (2009).

14. Vo-Dinh T, Wang HN, Scaffidi J. Plasmonic nanoprobes for SERS biosensing and bioimaging. J. Biophotonics 3, 89-102 (2010).

15. Vo-Dinh T, Fales AM, Griffin GD et al. Plasmonic nanoprobes: from chemical sensing to medical diagnostics and therapy. Nanoscale 5, 10127-10140 (2013).

16. Vo-Dinh T, Liu Y, Fales AM et al. SERS nanosensors and nanoreporters: golden opportunities in biomedical applications. Wiley Interdiscip. Rev. Nanomed. Nanobiotechnol. 7, 17-33 (2015). 
17. Wang HN, Crawford BM, Fales AM, Bowie BL, Seewaldt VL, Vo-Dinh T. Multiplexed detection of microRNA biomarkers using SERS-based inverse molecular sentinel (iMS) nanoprobes. J. Phys. Chem. C 120(37), 21047-21055 (2016).

18. Yuan H, Fales AM, Vo-Dinh T. TAT peptide-functionalized gold nanostars: enhanced intracellular delivery and efficient NIR photothermal therapy using ultralow irradiance. J. Am. Chem. Soc. 134(28), 11358-11361 (2012).

19. Liu Y, Ashton JR, Moding EJ et al. A plasmonic gold nanostar theranostic probe for in vivo tumor imaging and photothermal therapy. Theranostics 5(9), 946-960 (2015).

20. Yuan H, Khoury CG, Wilson CM, Grant GA, Bennett AJ, Vo-Dinh T. In vivo particle tracking and photothermal ablation using plasmon-resonant gold nanostars. Nanomedicine 8(8), 1355-1363 (2012).

21. Liu Y, Maccarini P, Palmer GM et al. Synergistic Immuno Photothermal Nanotherapy (SYMPHONY) for the treatment of unresec-table and metastatic cancers. Sci. Rep. 7, 8606 (2017).

22. Khoury CG, Vo-Dinh T. Gold nanostars for surface-enhanced raman scattering: synthesis, characterization and optimization. J. Phys. Chem. C Nanomater. Interfaces 2008(112), 18849-18859 (2008).

23. Yuan H, Khoury CG, Hwang H, Wilson CM, Grant GA, Vo-Dinh T. Gold nanostars: surfactant-free synthesis, 3D modelling, and two-photon photoluminescence imaging. Nanotechnology 23(7), 075102 (2012).

24. Norton SJ, Vo-Dinh T. Photothermal effects of plasmonic metal nanoparticles in a fluid. J. Appl. Phys. 119(8), 083105 (2016).

25. Yuan H, Gomez JA, Chien JS et al. Tracking mesenchymal stromal stem cells using TAT peptide-functionalized plasmonic gold nanoprobes. J. Biophotonics 9(4), 406-413 (2016).

26. Shammas RL, Fales AM, Crawford BM et al. Human adipose-derived stem cells labeled with plasmonic gold nanostars for cellular tracking and photothermal cancer cell ablation. Plast. Reconstr. Surg. 139, e900-e910 (2017).

27. Vinogradov SV, Bronich TK, Kabanov AV. Nanosized cationic hydrogels for drug delivery: preparation, properties and interactions with cells. Adv. Drug Deliv. Rev. 54, 135-147 (2002).

28. Ilium L, Davis SS, Wilson CG, Thomas NW, Frier M, Hardy JG. Blood clearance and organ deposition of intravenously administered colloidal particles. The effects of particle size, nature and shape. Int. J. Pharm. 12, 135-146 (1982).

29. Yuan H, Wilson CM, Xia J et al. Plasmonics-enhanced and optically modulated delivery of gold nanostars into brain tumor. Nanoscale 6(8), 4078-4082 (2014).

30. Falk MH, Issels RD. Hyperthermia in oncology. Int. J. Hyperthermia 17(1), 1-18 (2001).

31. Huang X, El-Sayed MA. Plasmonic photo-thermal therapy (PPTT). Alexandria J. Med. 47(1), 1-9 (2011).

32. Takada T, Yamashita T, Sato M et al. Growth inhibition of re-challenge B16 melanoma transplant by conjugates of melanogenesis substrate and magnetite nanoparticles as the basis for developing melanoma-targeted chemo-thermo-immunotherapy. J. Biomed. Biotechnol. 2009, 457936 (2009).

33. Koning GA, Eggermont AMM, Lindner LH, Hagen TLM. Hyperthermia and thermosensitive liposomes for improved delivery of chemotherapeutic drugs to solid tumors. Pharm. Res. 27(8), 1750-1754 (2010).

34. Wang C, Xu L, Liang C, Xiang J, Zhuang RP. Immunological responses triggered by photothermal therapy with carbon nanotubes in combination with anti-CTLA-4 therapy to inhibit cancer metastasis. Adv. Mater. 26(48), 8154-8162 (2014).

35. Kennedy LC, Bickford LR, Lewinski NA et al. A new era for cancer treatment: gold-nanoparticle-mediated thermal therapies. Small 7(2), 169-183 (2011).

36. Slovak R, Ludwig JM, Gettinger SN, Herbst RS, Kim HS. Immuno-thermal ablations - boosting the anticancer immune response. J. Immunother. Cancer 5, 78 (2017).

37. Wang C, Xu L, Liang C, Xiang J, Peng R, Liu Z. Immunological responses triggered by photothermal therapy with carbon nanotubes in combination with anti-CTLA-4 therapy to inhibit cancer metastasis. Adv. Mater. 26(48), 8154-8162 (2014).

38. Garg AD, Nowis D, Golab J, Vandenabeele P, Krysko DV, Agostinis P. Immunogenic cell death, DAMPs and anticancer therapeutics: an emerging amalgamation. Biochim. Biophys. Acta 805(1), 53-71 (2010).

39. Evans SS, Repasky EA, Fisher DT. Fever and the thermal regulation of immunity: the immune system feels the heat. Nat. Rev. Immunol. 15, 335-349 (2015).

40. Chen DS, Mellman I. Oncology meets immunology: the cancer-immunity cycle. Immunity 39(1), 1-10 (2013).

41. Pardoll DM. The blockade of immune checkpoints in cancer immunotherapy. Nat. Rev. Cancer 12, 252-264 (2012).

42. Inman BA, Sebo TJ, Frigola X et al. PD-L1 (B7-H1) expression by urothelial carcinoma of the bladder and BCG-induced granulomata: associations with localized stage progression. Cancer 109(8), 1499-1505 (2007).

43. Bellmunt J, Mullane SA, Werner L et al. Association of PD-L1 expression on tumor-infiltrating mononuclear cells and overall survival in patients with urothelial carcinoma. Ann. Oncol. 26(4), 812-817 (2015).

44. Inman BA, Frigola X, Dong H, Kwon ED. Costimulation coinhibition and cancer. Curr. Cancer Drug Targets 7(1), 15-30 (2007).

45. Inman BA, Longo TA, Ramalingam S, Harrison MR. Atezolizumab: a PD-L1-blocking antibody for bladder cancer. Clin. Cancer Res. 23(8), 1886-1890 (2017). 
46. Toraya-Brown S, Sheen MR, Zhang P et al. Local hyperthermia treatment of tumors induces CD8(+) T cell-mediated resistance against distal and secondary tumors. Nanomed. Nanotechnol. Biol. Med. 10, 1273-1285 (2014). 